\title{
Transmission USAgE AND Cost Allocation Using Shapley VALUE AND Tracing Method: A COMPARISON
}

\author{
Pawan Rathore ${ }^{1}$, Ganga Agnihotri ${ }^{2}$, Baseem $\mathrm{Khan}^{3}$ and Garima Naidu ${ }^{4}$ \\ 1,2,3,4 Department of Electrical Engineering, MANIT, Bhopal, India
}

\begin{abstract}
In the deregulated power system, transmission pricing has become a very important task because it is necessary to develop an efficient, feasible and reliable pricing scheme that can generate the useful economic signals to network users such as generating companies, transmission companies, distribution companies and customers. The objective of this paper is to compare transmission usage and cost allocation scheme to loads (and/or generators) based on Shapley value method and power flow tracing method. Modified Kirchhoff matrix is used for power flow tracing. A comparison is done between the both methods. A case study based on sample 6 bus power system is applied to check the feasibility and reliability of the proposed usage and cost allocation methodology.
\end{abstract}

\section{KEYWORDS}

Power Flow, Proportional Sharing Method, Shapley Value Method, Tracing Method, Transmission Usage Allocation, Transmission Cost Allocation, Transmission Pricing

\section{INTRODUCTION}

In recent years, due to deregulation and restructuring the Electric Supply Industries (ESIs) had to face major changes in its organization and structure. Deregulations have introduced operational independence and flexibility but in other hand it has also introduced complexity in functional and economic decision making. Transmission usage and cost allocation is one of such issues. In a pool based market or bilateral contract market a number of techniques have been proposed to allocate the power and cost of the transmission system to generators and/or loads. Various methods used for transmission usage allocation are as follows [1-3]:

- Pro rata method

- Proportional sharing method

- Equivalent bilateral exchanges

- Z bus method

- Nodal method

- Cooperative game theory

- Relative electrical distance

- Marginal participation method

The cost of the transmission system includes the cost of planning, operation and maintenance of the transmission corridor. It is the responsibility of the transmission users (generators and loads) to pay the transmission usage cost. In an open access market, it is very difficult to find a reliable 
and efficient method for allocating the usage and cost of the transmission system to its users [4]. Electric utilities traditionally allocate the transmission cost to each generator and load based on Postage Stamp and Contract Path methods [5]. In the Postage Stamp method, transmission network users are charged based on an average cost and the magnitude of the allocated power. On the other hand, in the Contract Path method, power is confined to flow along an artificially specified path. Based on the calculation of the actual extent of use of the transmission network MW Mile method is proposed [6], [7]. The cost depends upon the magnitude, the path and the distance travelled by the transacted power. Various modified MW Mile methodologies have been proposed in the literature [8-10].Tsukamoto and Iyoda [11] introduced the concept of cooperative game theory for fixed-cost allocation to wheeling transactions in a power system. Yu et al. [12] presented a method for transmission embedded cost allocation based on the use of line capacity. Tan and Lie [13] applied the Shapley value approach for the transmission network cost allocation. Zolezzi and Rudnick [14] allocated the cost of existing or expanding the network based on a model that integrates cooperation and coordination among the agents with solutions based on the Nucleolus and Shapley value approaches. Yu et al. [15] allocated the capacity-use and reliability-based transmission embedded cost using the Nucleolus and Shapley value concept. Stamtsis and Erlich [16] analyzed the cost allocation problem for the fixed cost of a power system and realized that the Shapley value is preferable when it lies in the core of the game [17]. In Aug 2013, Orfanos et al. [18] explained a power flow based method to allocate the transmission fixed cost in a pool based electricity market considering contingencies. They considered that the possible maximum used capacity of a transmission network is the maximum power flow during contingency analysis. The first attempt to trace real and reactive power flow was done by Bialek et al. [19] when Topological Generation Distribution factors based Power flow tracing were proposed in March 1996 which explained the method for tracing generators' output. Proportional Sharing method was used to trace the flow of electricity. Distribution factors [20] are defined by sensitivity analysis relating a change in power injection at a certain bus to a change in the power flow on a particular line. In 1996, Bialek [20] presented a method which allows allocating the supplement charge for transmission services to individual load or generator. Topological factor represents the share of the load in a power flow while the generalized factor shows the impact of the load on the power flow. Generalized Generation / Load Distribution Factors (GGDFs/GLDFs) are dependent upon line parameter not on the reference bus position. In Feb 1997, Kirschen et al. [21] introduced a power flow tracing method based on the proportional sharing assumption which introduced the concept of domains, commons, and links. In Nov 2000, Gubina et al. [22] presented a new method to determine the generators' contribution to a particular load by using the nodal generation distribution factors (NGDF-s). The method also handled the reactive power. In Aug 2000, Felix et al. [23] proposed the use of graph theory to calculate the contributions of individual generator and load to line flows and the real power transfer between individual generator and load. A matrix inverse calculation is required which is a time taking process for a large power system. In 2008, Xie et al. [24] proposed and explained the power flow tracing algorithms found in the Extended Incidence Matrix (EIM) considering loop flows. Charges had been allocated to generators and loads in 50:50 ratios. In Feb 2007, Conejo et al. [25] proposed a method of network cost allocation based on Z-bus matrix. In Aug 2006, Abhyankar et al. [26] proposed real power flow tracing method based on linear constrained optimization approach. They introduced a modified postage stamp method which evaluates a traceable solution that minimizes overall deviation from the postage stamp allocation. In Aug 2010, Rao et al. [27] explained the Min-Max fair allocation criteria for transmission system usage allocation.

In 2004, P. N. Biskas et al. [28] proposed a security constrained optimal power flow (SC-OPF) solution to trace each user's contribution to the line flows of the network. For this, first usage and then TRM allocation was done. In 1998, Silva et al. [29] considered the transmission network operation under normal as well as contingency condition for allocating cost to generators. In July 2004, D. Hur et al. [30] proposed various methods to allocate reliability contribution to market 
participants. In June 2010, V. Vijay et al [31] proposed a novel probabilistic transmission pricing methodology with consideration of transmission reliability margin. In 2008, H. Monsef et al. [32] presented the transmission cost allocation based on use of reliability margin under contingency condition. For this purpose a probability index was defined. The cost of the unused facility under normal system operation, i.e. the reliability margin cost has been proposed in [33- 35] to be allocated to transmission users following a contingency analysis.

In this paper, authors has presented a technique for allocating the usage and cost of the transmission system based on Shapley Value and power flow tracing method (Proportional Sharing). Different recovery policies for allocating the usage and cost are as follows:-

- Allocating $100 \%$ usage and cost to all loads.

- Allocating $100 \%$ usage and cost to all generators.

- Allocating 50\% - 50\% usage and cost to all generators and loads respectively.

- Allocating 33\% - 67\% usage and cost to all generators and loads respectively.

- Allocating $23 \%$ - $77 \%$ usage and cost to all generators and loads respectively.

In this paper, authors has considered $100 \%$ usage and cost allocation to all loads only. A comparison is done between the contributions of the loads to the usage and cost allocation based on both the methods. Cost allocation by Shapley Value method has been already done [13]. In this paper, usage allocation is done by Shapley Value Method and it is compared with that of power flow tracing method [10].

\section{Shapley Value Method}

The Shapley Value Method distributes the common usage and cost of cooperation by assuming that the usage and cost proportion of a participant in a coalition is determined by the incremental usage and cost that the participant generates by joining the coalition [36]. A cooperative game is defined as a game in which the players can conclude a contractual agreement as to which outcome will be chosen to exploit the possibility of common interests [11]. That is why transmission usage and cost allocation problem can be defined as cooperative game and it is easy to be applied.

The Shapley Value is calculated as follows. Let $c$ be the characteristic function and $i$ be any player in the game. It is assumed that the cost of serving none to be zero, that is, $c(0)=0$. Therefore, the allocation $a_{i}$ to player $i$ by the Shapley Value method is determined by

$$
a_{i}=\sum_{S}\left[\frac{(|S|-1) ! *(n-|S|) !}{n !} *[c(S)-c(S-\{i\})]\right]
$$

where $S$ represents the number of players in the coalition containing $i, n$ represents the total number of players in the game.

The term $[c(S)-c(S-\{i\})]$ calculates the incremental contribution that player $i$ makes to coalition $S$. $n$ ! determines the coalition permutations that can be created from the participants in the grand coalition. There are $(|S|-1)$ ! different orders in which the first $(|S|-1)$ players can precede $i$, and $(n-|S|)$ ! different orders in which the remaining $(n-|S|)$ players can follow, for 
a total of $(|S|-1) !(n-|S|)$ ! permutations in which the players precede $i$ precisely [9]. Taken together, the expression $[(|S|-1) ! /(n-|S|) !] \div n !]$ is a weighting factor that assigns equal share of the marginal contribution generated to each coalition of interest. The contribution of the player $i$ is thus weighted and summed for all coalitions where $i$ appears in $S$. The end result of the process is that each player $i$ is allotted a value equal to its expected marginal contribution (or incremental value) [13].

The characteristic function $c(S)$ of the proposed cooperative game can be calculated as follows:

$$
\begin{gathered}
c(S)=\sum_{l \in N_{L}}\left(P_{m-n}\right) \\
c(S)=\sum_{l \in N_{L}}\left(P_{m-n} * C_{m-n}\right)
\end{gathered}
$$

where $c(S)$ is the fixed cost of providing transmission service to coalition $S, P_{m-n}$ is the power flow in the line $m-n$ and $C_{m-n}$ is the cost of the line $m-n$. Figure 1. shows the process chart for the determination of transmission charges.

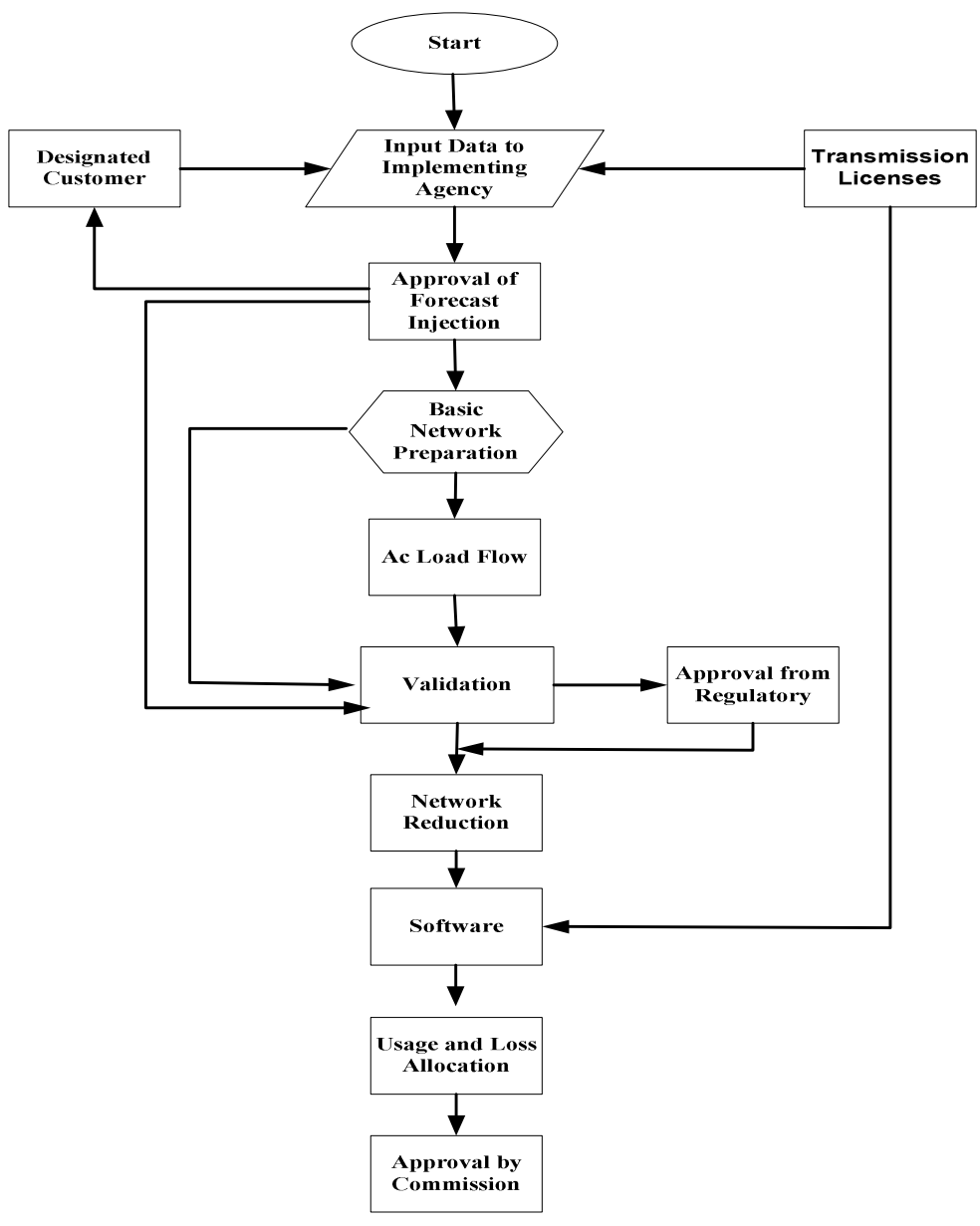

Figure 1. Process chart for the determination of transmission charges. 


\section{Power Flow Tracing Method}

In many literatures, power flow tracing method is referred for the calculation of the usage of the branch in the power system. Proportional Sharing principle is the basic assumption used by the power flow tracing method. The transmission usage and cost calculated by the power flow tracing method are obtained observing how the power injection into the nodes distributes itself through the transmission lines [4]. According to the power flow tracing method, each injection of power at a bus has a direct relationship with each extraction of power at this bus. To allocate the power two algorithms had been proposed: first upstream looking algorithm i.e. usage allocated to generators and, second downstream looking algorithm i.e. usage allocated to loads. Proportional Sharing principle is illustrated in Figure 2.

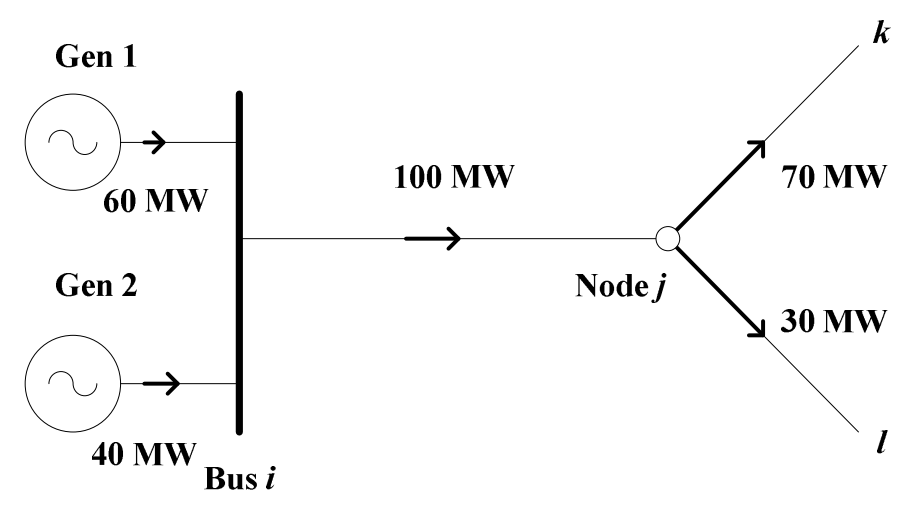

Figure 2. Proportional Sharing Method.

The line $i-j$ power inflow through node $j$ is $P_{j}=100 \mathrm{MW}$, of which $60 \%$ is assumed to be supplied by Gen 1 and rest $40 \%$ by Gen 2 . Hence the 70MW outflow of the line $j-k$ is allocated to Gen 1 as $70 \times 60 \%=42 \mathrm{MW}$ and to Gen 2 as $70 \times 40 \%=28 \mathrm{MW}$. Similarly the $30 \mathrm{MW}$ outflow of the line $j-l$ is allocated to Gen 1 as $30 \times 60 \%=18 \mathrm{MW}$ and to Gen 2 as $30 \mathrm{x}$ $40 \%=12 \mathrm{MW}$. Thus, for proportional sharing method, important data required are the proportion of branch inflow in the node and the proportion of branch outflow in the node. Branch inflow is defined as branch flow entering a node and similarly branch outflow is defined as branch flow leaving a node.

\subsection{Upstream Looking Algorithm}

In the upstream looking algorithm, using Modified Kirchhoff Matrix [10] the transmission usage of a transmission line is allocated to the generators. The generator share situated at bus $i$ to the line $m-n$ through path $k$ can be calculated using the following equation:

$$
P_{G i \rightarrow m-n}^{k}=t_{i m} P_{m-n} a_{g}
$$

where $t_{i m}$ is the supply factor matrix of the generator situated at bus $i$ to the line $m-n, P_{m-n}$ is the power flow in the line $m-n, P_{G i \rightarrow m-n}^{k}$ is the $i^{t h}$ generator share, $a_{g}$ is the percentage of the allocation. 
The total generator share situated at bus $i$ to the line $m-n$ through all paths can be calculated using the following equation:

$$
P_{G i \rightarrow m-n}=\sum_{k \in N_{p i \rightarrow m-n}} P_{G i \rightarrow m-n}^{k}
$$

where $N_{p i \rightarrow m-n}$ is the total number of path, $P_{G i \rightarrow m-n}$ is the total generator share situated at bus $i$. The generators' share to loads in the system can be calculated using the following equation:

$$
P_{G i}=\sum_{j=1}^{n} t_{i-j} P_{L j}
$$

where $t_{i-j} P_{L j}$ denotes the active power distribution of generation output at bus $i$ to the load situated at bus $j[23]$. From Eq. (6),

$$
P_{G i-j}=t_{i-j} P_{L j}
$$

Thus, Eq. (7) gives the generators' share to loads in the system.

The cost paid by generator $i$ for using transmission line $m-n$ can be calculated using the following equation:

$$
T C_{G i \rightarrow m-n}=C_{m-n} * P_{G i \rightarrow m-n}
$$

where $C_{m-n}$ is the cost of line $m-n, T C_{G i \rightarrow m-n}$ is the transmission cost of the line $m-n$ due to generator $i$.

The cost paid by generator $i$ for using transmission system can be calculated using the following equation:

$$
T C_{G i}=\sum_{k \in N_{L}} T C_{G i \rightarrow m-n}
$$

where $N_{L}$ is the total number of lines, $T C_{G i}$ is the total transmission cost of the all lines due to generator $i$.

\subsection{Downstream Looking Algorithm}

The load share situated at bus $i$ to the line $m-n$ through path $k$ can be calculated using the following equation:

$$
P_{L i \rightarrow m-n}^{k}=t_{i m} P_{m-n} a_{l}
$$

$P_{L i \rightarrow m-n}^{k}$ is the $i^{t h}$ generator share, $a_{l}$ is the percentage of the allocation.

The total load share situated at bus $i$ to the line $m-n$ through all paths can be calculated using the following equation:

$$
P_{L i \rightarrow m-n}=\sum_{k \in N_{p i \rightarrow m-n}} P_{L i \rightarrow m-n}^{k}
$$


$P_{L i \rightarrow m-n}$ is the total load share situated at bus $i$.

The loads' share to generators in the system can be calculated using the following equation:

$$
P_{L i}=\sum_{j=1}^{n} t_{i-j} P_{G j}
$$

where $t_{i-j} P_{G j}$ denotes the active power distribution of load at bus $i$ to the generator situated at bus $j[23]$.

$$
P_{L i-j}=t_{i-j} P_{G j}
$$

Thus (13) gives the loads' share to generators in the system.

The cost paid by loads $i$ for using transmission line $m-n$ can be calculated using the following equation:

$$
T C_{L i \rightarrow m-n}=C_{m-n} * P_{L i \rightarrow m-n}
$$

where $C_{m-n}$ is the cost of line $m-n, T C_{L i \rightarrow m-n}$ is the transmission cost of the line $m-n$ due to load $i$.

The cost paid by load $i$ for using transmission system can be calculated using the following equation:

$$
T C_{L i}=\sum_{k \in N_{L}} T C_{L i \rightarrow m-n}
$$

where $N_{L}$ is the total number of lines, $T C_{L i}$ is the total transmission cost of the all lines due to load $i$.

Figure 3. shows the usage cost allocation by Transmission System Operator (TSO).

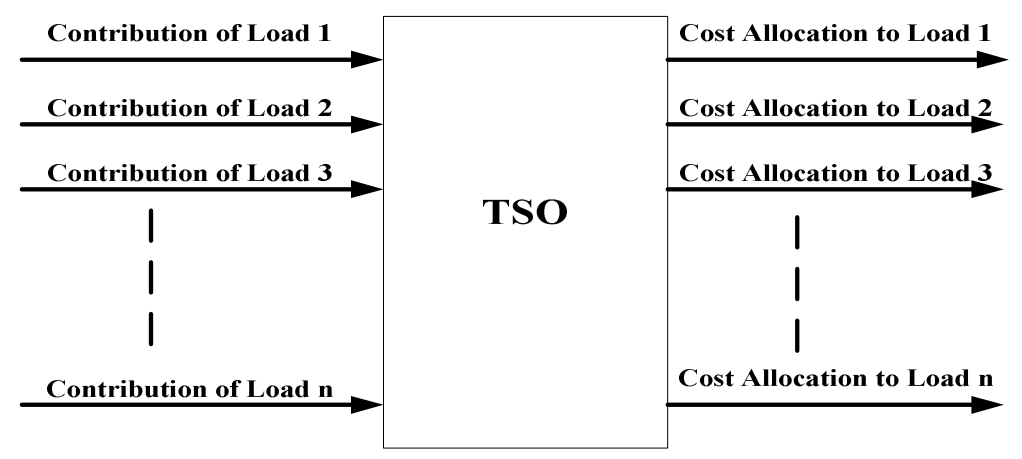

Figure 3. Usage Cost Allocation by TSO [13].

\section{CaSe Study}

Figure 4 depicts a 6 bus test system [40], which is composed of three loads and three generators. The system data is given in Table. In an open access transmission system, it is supposed that three independent generators Gen 1, Gen 2 and Gen 3 sell their electricity to three loads Load 4, Load 5 
and Load 6. In the deregulated market Transmission System Operator (TSO) is responsible for providing the required transmission service and allocating the cost incurred to the loads involved in the service [38]. It is assumed here that the cost for all the transmission lines is equivalent to its impedance of the lines. Table 1. shows the transaction details of the 6 bus power system.

\subsection{Shapley Value Method}

Let $P=\{1,2,3\}$ represent the set of players in the game, in which elements 1,2 and 3 represent Load 4, Load 5 and Load 6 respectively. In cooperative game theory for $n$ players, the number of coalitions will be $2^{n}-1$. Thus, for the above power system the number of coalitions will be 7 which are as follows:

$C=(\{1\},\{2\},\{3\},\{1,2\},\{1,3\},\{2,3\},\{1,2,3\})$ denotes all the possible coalitions between these three set of players.

Table 1. Transaction Details of the 6 Bus System

\begin{tabular}{|l|l|l|l|l|}
\hline Transactions & Gen_bus & Load_bus & $\begin{array}{l}\mathbf{P}_{\mathbf{L}} \\
(\mathbf{M W})\end{array}$ & $\begin{array}{l}\mathbf{Q}_{\mathbf{L}} \\
(\mathbf{M V A r})\end{array}$ \\
\hline 1 & 1 & 5 & 70 & 70 \\
\hline 2 & 2 & 4 & 70 & 70 \\
\hline 3 & 3 & 6 & 70 & 70 \\
\hline
\end{tabular}

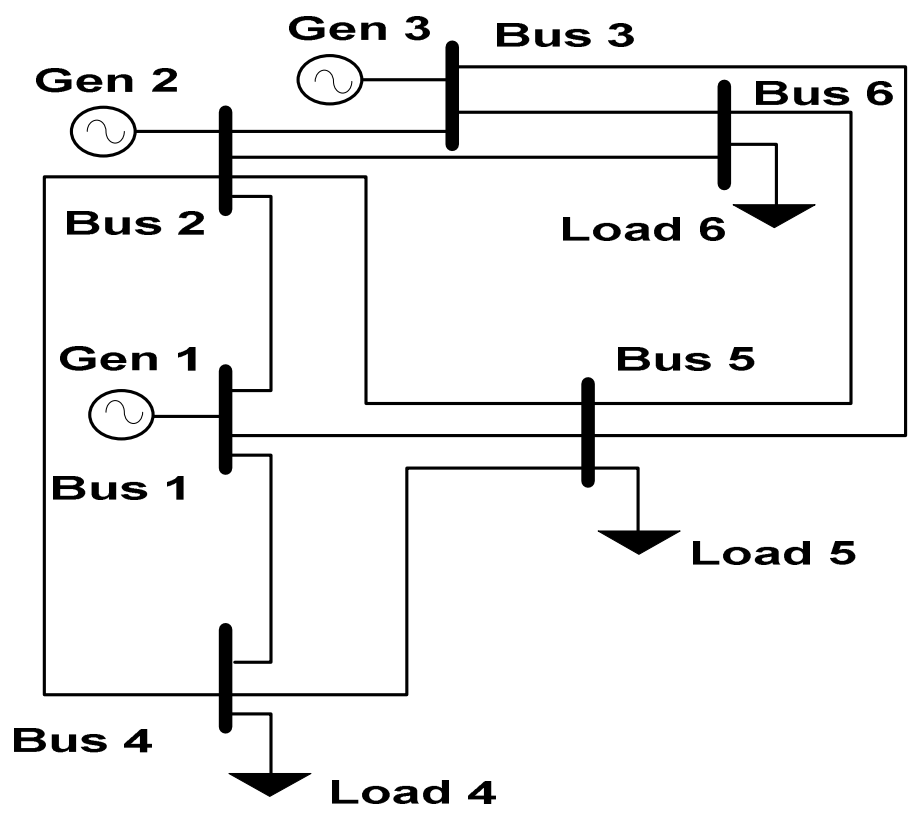

Figure 4. Sample 6 Bus System.

The optimal power flow is then performed by the TSO to determine the distribution of power flow through the whole system in the case of each coalition while considering the physical constraints into account which is given in Table 2. Figure 5. shows the power flow in the lines. 
Electrical and Electronics Engineering: An International Journal (ELELIJ) Vol 3, No 3, August 2014

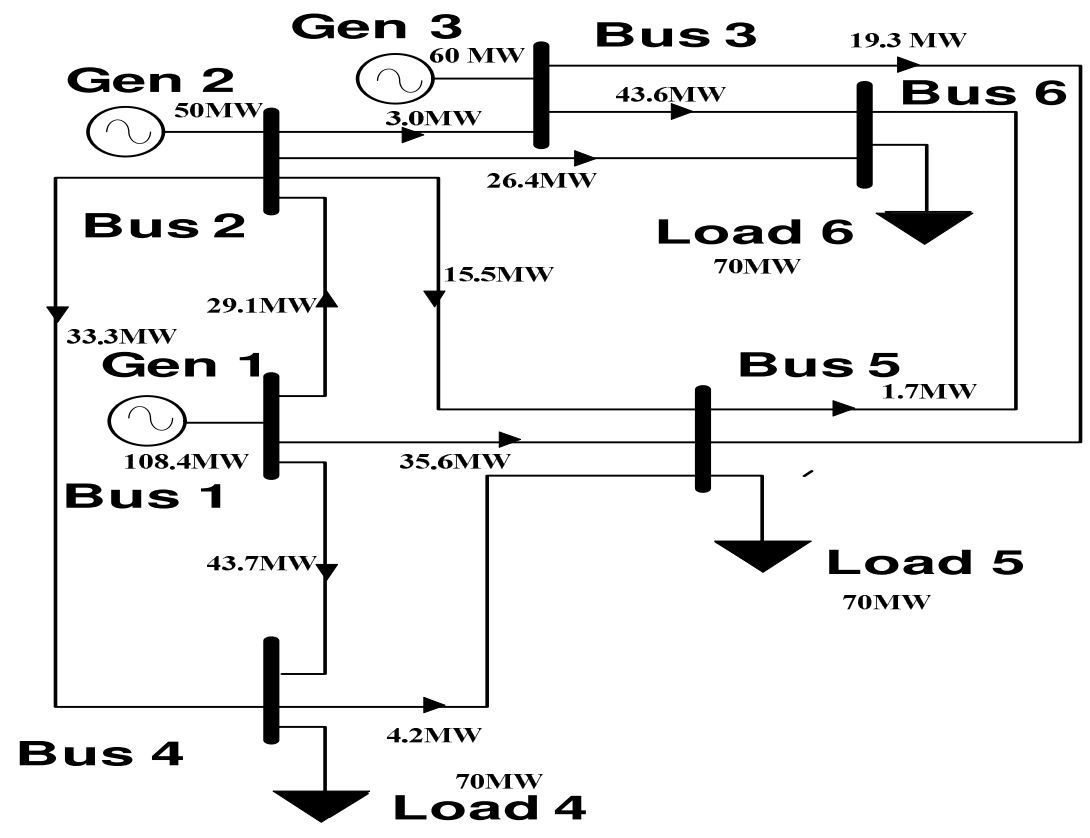

Figure 5. Power Flow of the Sample 6 Bus System.

Table 2. Characteristic Function Usage Values for Loads

\begin{tabular}{|l|l|l|l|l|l|l|l|}
\hline \multirow{2}{*}{ Line } & \multicolumn{6}{|c|}{ Different coalition for usage allocation (MW) } \\
\cline { 2 - 8 } & $\{\mathbf{1}\}$ & $\{\mathbf{2}\}$ & $\{\mathbf{3}\}$ & $\{\mathbf{1 , 2}\}$ & $\{\mathbf{1 , 3}\}$ & $\{\mathbf{2 , 3}\}$ & $\{\mathbf{1 , 2 , 3}\}$ \\
\hline $1-2$ & 21.76 & 22.07 & 17.14 & 0.09 & 4.93 & 5.10 & 29.12 \\
\hline $1-4$ & 1.88 & 14.06 & 12.69 & 21.57 & 22.67 & 8.12 & 43.70 \\
\hline $1-5$ & 15.64 & 0.22 & 8.18 & 13.68 & 6.10 & 21.13 & 35.63 \\
\hline $2-3$ & 18.66 & 9.57 & 4.04 & 10.96 & 5.50 & 4.33 & 2.98 \\
\hline $2-4$ & 57.43 & 22.09 & 12.97 & 50.19 & 41.07 & 4.87 & 33.28 \\
\hline $2-5$ & 1.35 & 16.88 & 5.08 & 14.39 & 2.33 & 18.05 & 15.49 \\
\hline $2-6$ & 12.43 & 2.03 & 18.52 & 3.53 & 17.00 & 27.82 & 26.43 \\
\hline $3-5$ & 18.79 & 29.97 & 10.58 & 28.64 & 9.12 & 20.62 & 19.33 \\
\hline $3-6$ & 22.38 & 20.39 & 45.35 & 20.33 & 45.34 & 43.66 & 43.62 \\
\hline $4-5$ & 13.11 & 7.69 & 0.11 & 0.82 & 8.21 & 12.95 & 4.21 \\
\hline $5-6$ & 9.67 & 17.78 & 7.38 & 16.19 & 8.90 & 0.22 & 1.71 \\
\hline
\end{tabular}

When there is no cooperation, i.e. the transmission system is used exclusively by each player, the value of the characteristic function in Eq. (2) mentioned above for specific coalition $\{1\}$, $\{2\}$ and $\{3\}$ is as follows:

$$
\begin{aligned}
t u(\{1\}) & =193.115 M W \\
t u(\{2\}) & =162.757 M W \\
t u(\{3\}) & =142.016 M W
\end{aligned}
$$

where $\operatorname{tu}(\{1\})$ represents the transmission usage allocation involved when the transaction 1 alone present in the system between gen_bus 1 and load_bus 5 operating. 
However, if more than one player agrees to use the transmission system simultaneously, the power flow through some lines would drop due to the possible counter-flow which relieves the congestion. In this situation, the characteristic function and its value for coalition $\{1,2\},\{1,3\},\{2,3\}$ is as follows:

$$
\begin{gathered}
t u(\{1,2\})=180.379 M W \\
t u(\{1,3\})=171.175 M W \\
t u(\{2,3\})=166.886 M W
\end{gathered}
$$

where $\operatorname{tu}(\{1,2\})$ represents the transmission usage allocation involved when the transaction operating together between gen_bus 1 and load_bus 5 along with gen_bus 2 and load_bus 4 operating and so on.

Furthermore, the characteristic function of the grand coalition $\{1,2,3\}$ is as follows:

$$
\operatorname{tu}(\{1,2,3\})=255.496 M W
$$

where $t u(\{1,2,3\})$ represents the transmission usage allocation involved when the transaction operating together between gen_bus 1 and load_bus 5 along with gen_bus 2 and load_bus 4 and gen_bus 3 and load_bus 6 operating and so on. It is very obvious that the total transmission usage allocation in cooperation is much less than when the system is occupied monopolistically by load. Now the problem is how to distribute the transmission usage according to each player's incremental effect to the coalition. To find only one optimal solution, the Shapley Value concept would be used.

Let $u a_{i}$ denote the usage allocated to player $i$ by the Shapley Value. Thus $u a_{i}$ is calculated as:

$$
\begin{aligned}
u a_{1}= & \frac{0 ! * 2 !}{3 !}[t u(\{1\})-t u(\{1\}-\{1\})]+\frac{1 ! * 1 !}{3 !}[t u(\{1,2\})-t u(\{1,2\}-\{1\})]+ \\
& \frac{1 ! * 1 !}{3 !}[t u(\{1,3\})-t u(\{1,3\}-\{1\})]+\frac{2 ! * 0 !}{3 !}[t u(\{1,2,3\})-t u(\{1,2,3\}-\{1\})] \\
u a_{1}= & \frac{0 ! * 2 !}{3 !}[t u(\{1\})-0]+\frac{1 ! * 1 !}{3 !}[t u(\{1,2\})-t u(\{2\})]+\frac{1 ! * 1 !}{3 !}[t u(\{1,3\})-t u(\{3\})]+ \\
& \frac{2 ! * 0 !}{3 !}[t u(\{1,2,3\})-t u(\{2,3\})] \\
u a_{1}= & \frac{1}{3}[193.115-0]+\frac{1}{6}[180.379-162.757]+\frac{1}{6}[171.175-142.016]+\frac{1}{3}[255.496-166.886] \\
u a_{1}= & 101.705 \mathrm{MW}
\end{aligned}
$$

Similar allocation may be calculated for other transactions. The transmission usage allocation of transactions 2 and 3 can easily be calculated as:

$$
u a_{2}=84.382 M W \quad u a_{3}=69.41 M W
$$

It could be observed that the transmission usage allocation using the Shapley Value met the following rational conditions: 


\subsubsection{Coalition Rationality}

Coalition rationality which requires that no player would be allocated that is greater than what it would be to serve that player alone (i.e., its stand alone). From the above case, we could find this requirement is met by the following:

$$
\begin{aligned}
& u a_{1}=101.705 \leq t u(\{1\})=193.115 \\
& u a_{2}=84.382 \leq t u(\{2\})=162.757 \\
& u a_{3}=69.41 \leq t u(\{3\})=142.016
\end{aligned}
$$

\subsubsection{Individual Rationality}

Individual rationality which requires that no player would be allocated less than its marginal usage.

$$
u a_{1}+u a_{2}=186.087 \geq[t u(\{1,2,3\})-t u(\{3\})]=113.48
$$

\subsubsection{Global Rationality}

Global rationality that is the usage should be allocated exactly as follows:

$$
u a_{1}+u a_{2}+u a_{3}=t u(\{1,2,3\})=255.496
$$

From these results, the benefit of cooperation between the users of the transmission network is evident. The TSO thus would encourage the load to take this opportunity. Similarly, different coalition for transmission usage cost allocation for sample 6 bus system is shown as:

For transmission usage cost allocation the value of the characteristic function in Eq. (3) mentioned above for specific coalition $\{1\},\{2\}$ and $\{3\}$ is as follows:

$$
\begin{aligned}
& t c(\{1\})=40936.3 \$ / h r \\
& t c(\{2\})=38303.4 \$ / h r \\
& t c(\{3\})=27030.1 \$ / h r
\end{aligned}
$$

where $\operatorname{tc}(\{1\})$ represents the transmission cost involved when the transaction 1 alone present in the system between gen_bus 1 and load_bus 5 operating.

However, if more than one player agrees to use the transmission system simultaneously, the power flow through some lines would drop due to the possible counter-flow which relieves the congestion. In this situation, the characteristic function and its value for coalition $\{1,2\},\{1,3\},\{2,3\}$ is as follows:

$$
\begin{aligned}
& t c(\{1,2\})=38175.3 \$ / h r \\
& t c(\{1,3\})=31725.4 \$ / h r \\
& t c(\{2,3\})=38848.7 \$ / h r
\end{aligned}
$$

where $t c(\{2,3\})$ represents the transmission cost involved when the transaction operating together between gen_bus 2 and load_bus 4 along with gen_bus 3 and load_bus 6 operating and so on.

Furthermore, the characteristic function of the grand coalition $\{1,2,3\}$ is as follows:

$t c(\{1,2,3\})=53969.5 \$ / h r$ 
where $\operatorname{tc}(\{1,2,3\})$ represents the transmission cost involved when the transaction operating together between gen_bus 1 and load_bus 5 along with gen_bus 2 and load_bus 4 and gen_bus 3 and load_bus 6 operating and so on.

It is very obvious that the total transmission usage cost in cooperation is much less than when the system is occupied monopolistically by load. Now the problem is how to distribute the transmission usage cost according to each player's incremental effect to the coalition. To find only one optimal solution, the Shapley Value concept would be used. Table 3. shows the different coalition for cost allocation to loads.

Table 3. Characteristic Function Cost Values for Loads

\begin{tabular}{|l|l|l|l|l|l|l|l|}
\hline \multirow{2}{*}{ Line } & \multicolumn{7}{|l|}{ Different coalition for cost allocation $\mathbf{( \$ / h r )}$} \\
\cline { 2 - 8 } & $\{\mathbf{1}\}$ & $\{\mathbf{2}\}$ & $\{\mathbf{3}\}$ & $\{\mathbf{1 , 2}\}$ & $\{\mathbf{1 , 3}\}$ & $\{\mathbf{2 , 3}\}$ & $\{\mathbf{1 , 2 , 3}\}$ \\
\hline $1-2$ & 4866.2 & 4934.7 & 3831.9 & 20.5 & 1102.4 & 1140.8 & 6511.0 \\
\hline $1-4$ & 387.9 & 2898.8 & 2615.7 & 4447.7 & 4674.0 & 1674.7 & 9008.3 \\
\hline $1-5$ & 4857.4 & 68.9 & 2539.3 & 4246.7 & 1892.6 & 6561.4 & 11063.3 \\
\hline $2-3$ & 4758.5 & 2440.8 & 1030.1 & 2793.1 & 1402.5 & 1103.1 & 760.8 \\
\hline $2-4$ & 6421.2 & 2469.8 & 1449.9 & 5611.2 & 4592.1 & 545.0 & 3720.7 \\
\hline $2-5$ & 426.5 & 5336.6 & 1605.7 & 4548.9 & 738.4 & 5708.8 & 4899.8 \\
\hline $2-6$ & 2634.0 & 429.9 & 3924.0 & 747.3 & 3601.3 & 5894.5 & 5600.2 \\
\hline $3-5$ & 5381.1 & 8582.8 & 3028.9 & 8201.3 & 2612.8 & 5905.0 & 5534.3 \\
\hline $3-6$ & 2282.1 & 2079.8 & 4624.7 & 2073.6 & 4623.5 & 4452.3 & 4448.1 \\
\hline $4-5$ & 5863.8 & 3438.0 & 47.2 & 366.0 & 3669.9 & 5792.1 & 1882.4 \\
\hline $5-6$ & 3057.7 & 5623.3 & 2332.8 & 5119.0 & 2815.9 & 70.9 & 540.6 \\
\hline
\end{tabular}

Let $c a_{i}$ denote the cost allocated to player $i$ by the Shapley Value. Thus $c a_{i}$ is calculated as:

$$
\begin{aligned}
c a_{1}= & \frac{0 ! * 2 !}{3 !}[t c(\{1\})-t c(\{1\}-\{1\})]+\frac{1 ! * 1 !}{3 !}[t c(\{1,2\})-t c(\{1,2\}-\{1\})]+ \\
& \frac{1 ! * 1 !}{3 !}[t c(\{1,3\})-t c(\{1,3\}-\{1\})]+\frac{2 ! * 0 !}{3 !}[t c(\{1,2,3\})-t c(\{1,2,3\}-\{1\})] \\
c a_{1}= & \frac{0 ! * 2 !}{3 !}[t c(\{1\})-0]+\frac{1 ! * 1 !}{3 !}[t c(\{1,2\})-t c(\{2\})]+\frac{1 ! * 1 !}{3 !}[t c(\{1,3\})-t c(\{3\})]+ \\
& \frac{2 ! * 0 !}{3 !}[t c(\{1,2,3\})-t c(\{2,3\})] \\
c a_{1}= & \frac{1}{3}[40936.3-0]+\frac{1}{6}[38175.3-38303.4]+\frac{1}{6}[31725.4-27030.1]+\frac{1}{3}[53969.5-38848.7] \\
c a_{1}= & 19446.9 \$ / h r
\end{aligned}
$$

Similar allocation may be calculated for other transactions. The usage cost allocation of transactions 2 and 3 can easily be calculated as:

$c a_{2}=21692.1 \$ / h r \quad c a_{3}=12830.5 \$ / h r$

Thus total cost allocation can be calculated as follows: 
$T c a=c a_{1}+c a_{2}+c a_{3}=53969.5 \$ / h r$

It could be observed that the transmission usage cost allocation using the Shapley Value met the all rational conditions just like transmission usage allocation met the all above rational conditions. The individual line allocations as well as the overall percentage allocations are presented in Table 4.

Table 4. Allocations Based On Shapley Value Calculations to Different Loads

\begin{tabular}{|l|l|l|l|}
\hline Line & L4 (\%) & L5 (\%) & L6 (\%) \\
\hline $1-2$ & 36.64 & 36.58 & 26.78 \\
\hline $1-4$ & 6.84 & 50.34 & 42.82 \\
\hline $1-5$ & 66.71 & 0.93 & 32.36 \\
\hline $2-3$ & 58.62 & 29.60 & 11.78 \\
\hline $2-4$ & 62.97 & 23.84 & 13.19 \\
\hline $2-5$ & 5.95 & 73.27 & 20.78 \\
\hline $2-6$ & 39.32 & 6.32 & 54.36 \\
\hline $3-5$ & 32.34 & 50.77 & 16.89 \\
\hline $3-6$ & 26.47 & 23.75 & 49.78 \\
\hline $4-5$ & 63.11 & 36.42 & 0.47 \\
\hline $5-6$ & 28.42 & 51.46 & 20.12 \\
\hline $\begin{array}{l}\text { Overall } \\
\text { Allocation }\end{array}$ & 39.81 & 33.03 & 27.17 \\
\hline
\end{tabular}

\subsection{Power Flow Tracing Method}

Table 5. shows that the total flows in the lines are fully extracted by the different loads. It can be seen that the power extracted by the Load 4 is lower than the others in the lines 3-5, 3-6 and 5-6. For the line 4-5, entire power is extracted by the Load 4 only. The flow in the lines 4-5 and 5-6 are extracted by a single Load i.e. Load 4 and Load 5 respectively. For the line 2-3, nearly equal power is extracted by the different loads. Table 6 . shows analysis of cost allocation to different loads under rated capacity.

Table 5. Analysis of Usage Allocation to Different Loads

\begin{tabular}{|l|l|l|l|l|}
\hline Line & $\begin{array}{l}\text { Flow } \\
(\mathbf{M W})\end{array}$ & $\begin{array}{l}\text { Extracted } \\
\text { by Load 4 } \\
(\mathbf{M W})\end{array}$ & $\begin{array}{l}\text { Extracted } \\
\text { by Load 5 } \\
\text { (MW) }\end{array}$ & $\begin{array}{l}\text { Extracted } \\
\text { by Load 6 } \\
\text { (MW) }\end{array}$ \\
\hline $1-2$ & 29.1 & 14.2 & 11.77 & 3.13 \\
\hline $1-4$ & 43.7 & 21.32 & 17.67 & 4.71 \\
\hline $1-5$ & 35.6 & 17.36 & 14.4 & 3.83 \\
\hline $2-3$ & 3 & 1.21 & 0.69 & 1.11 \\
\hline $2-4$ & 33.3 & 13.37 & 7.61 & 12.32 \\
\hline $2-5$ & 15.5 & 6.22 & 3.54 & 5.74 \\
\hline $2-6$ & 26.4 & 10.6 & 6.03 & 9.77 \\
\hline $3-5$ & 19.3 & 0 & 5.78 & 13.52 \\
\hline $3-6$ & 43.6 & 0 & 13.07 & 30.53 \\
\hline $4-5$ & 4.2 & 3.96 & 0.23 & 0.01 \\
\hline $5-6$ & 1.7 & 0 & 1.66 & 0.04 \\
\hline
\end{tabular}


Electrical and Electronics Engineering: An International Journal (ELELIJ) Vol 3, No 3, August 2014

Table 6. Analysis of Cost Allocation to Different Loads

\begin{tabular}{|c|c|c|c|c|}
\hline Line & $\begin{array}{l}\text { Cost of } \\
\text { Line } \\
\text { (\$/MWhr) }\end{array}$ & $\begin{array}{l}\text { Cost } \\
\text { Allocated } \\
\text { to Load } 4 \\
(\$ / h r)\end{array}$ & $\begin{array}{l}\text { Cost } \\
\text { Allocated } \\
\text { to Load } 5 \\
(\$ / h r)\end{array}$ & $\begin{array}{l}\text { Cost } \\
\text { Allocated to } \\
\text { Load } 6 \\
(\$ / h r)\end{array}$ \\
\hline $1-2$ & 223.607 & 3175.217 & 2631.852 & 699.8893 \\
\hline $1-4$ & 206.155 & 4395.231 & 3642.764 & 970.9915 \\
\hline $1-5$ & 310.485 & 5390.025 & 4470.988 & 1189.159 \\
\hline $2-3$ & 254.951 & 308.4907 & 175.9162 & 282.9956 \\
\hline $2-4$ & 111.803 & 1494.811 & 850.8239 & 1377.418 \\
\hline $2-5$ & 316.228 & 1966.937 & 1119.446 & 1815.148 \\
\hline $2-6$ & 211.896 & 2246.1 & 1277.734 & 2070.226 \\
\hline $3-5$ & 286.356 & 0 & 1655.14 & 3871.539 \\
\hline $3-6$ & 101.980 & 0 & 1332.884 & 3113.462 \\
\hline $4-5$ & 447.214 & 1770.966 & 102.8591 & 4.472136 \\
\hline $5-6$ & 316.228 & 0 & 524.9381 & 12.64911 \\
\hline
\end{tabular}

The cost paid by load $i\left(T C_{L i}\right.$ ) for using transmission system by power flow tracing method can be calculated using Eq. (14).

$T C_{L 1}=20747.8 \$ / h r T C_{L 2}=17785.3 \$ / h r \quad T C_{L 3}=15407.9 \$ / h r$

The total cost paid by all loads $\left(T C_{L}\right)$ for using transmission system by power flow tracing method is given as:

$T C_{L}=T C_{L 1}+T C_{L 2}+T C_{L 3}=53941.1 \$ / h r$

The individual line allocations as well as the overall percentage allocations using power flow tracing method are presented in Table 7. It can be seen that the contribution by the Load 4 is lower than the others in the lines 3-5, 3-6 and 5-6. The contribution of the lines 4-5 and 5-6 are due to a single Load i.e. Load 1 and Load 2 respectively.

Table 7. Allocations based on Tracing Method Calculations to Different Loads

\begin{tabular}{|l|l|l|l|}
\hline Line & L4 (\%) & L5 (\%) & L6 (\%) \\
\hline $1-2$ & 48.79 & 40.44 & 10.75 \\
\hline $1-4$ & 48.78 & 40.43 & 10.77 \\
\hline $1-5$ & 48.77 & 40.46 & 10.76 \\
\hline $2-3$ & 40.19 & 22.92 & 36.87 \\
\hline $2-4$ & 40.15 & 22.85 & 36.99 \\
\hline $2-5$ & 40.12 & 22.83 & 37.03 \\
\hline $2-6$ & 40.15 & 22.84 & 37.00 \\
\hline $3-5$ & 0 & 29.94 & 70.05 \\
\hline $3-6$ & 0 & 29.97 & 70.02 \\
\hline $4-5$ & 94.28 & 5.47 & 0.24 \\
\hline $5-6$ & 0 & 97.64 & 2.35 \\
\hline $\begin{array}{l}\text { Overall } \\
\text { Allocation }\end{array}$ & 34.55 & 32.28 & 33.17 \\
\hline
\end{tabular}


Electrical and Electronics Engineering: An International Journal (ELELIJ) Vol 3, No 3, August 2014

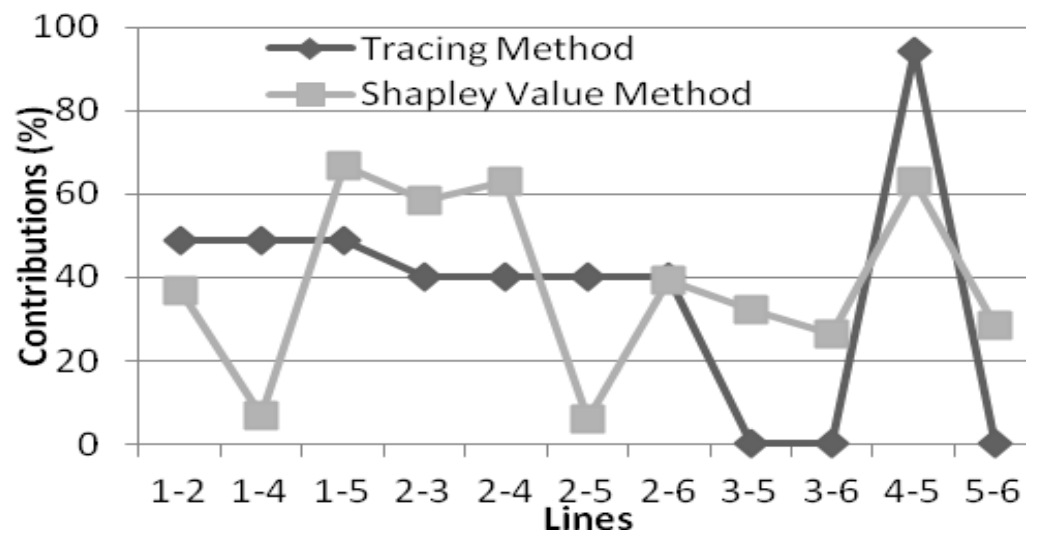

Figure 6 (a). Comparison of the usage allocation based on Tracing Method and Shapley Value Calculations to Load 4.

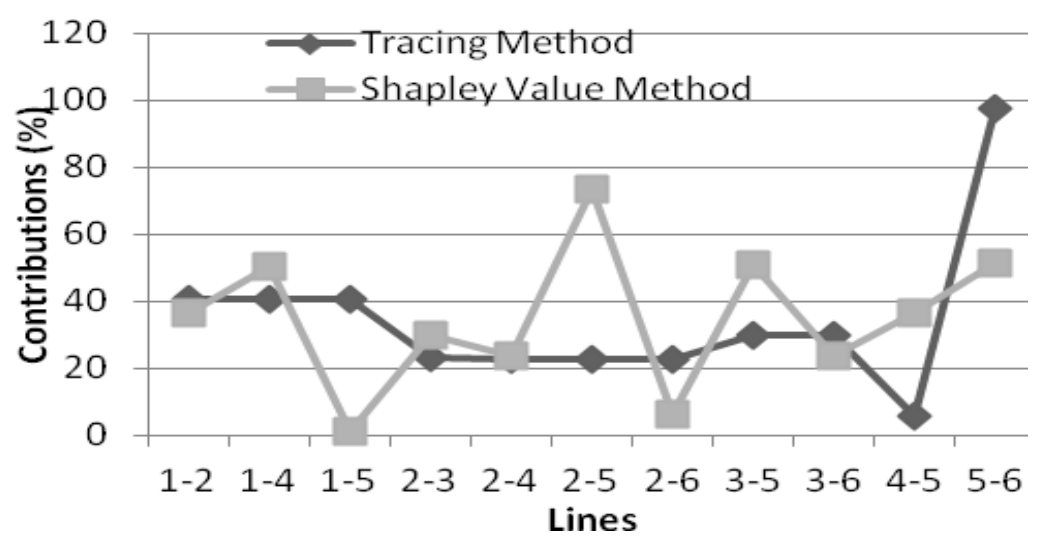

Figure 6 (b). Comparison of the usage allocation based on Tracing Method and Shapley Value Calculations to Load 5.

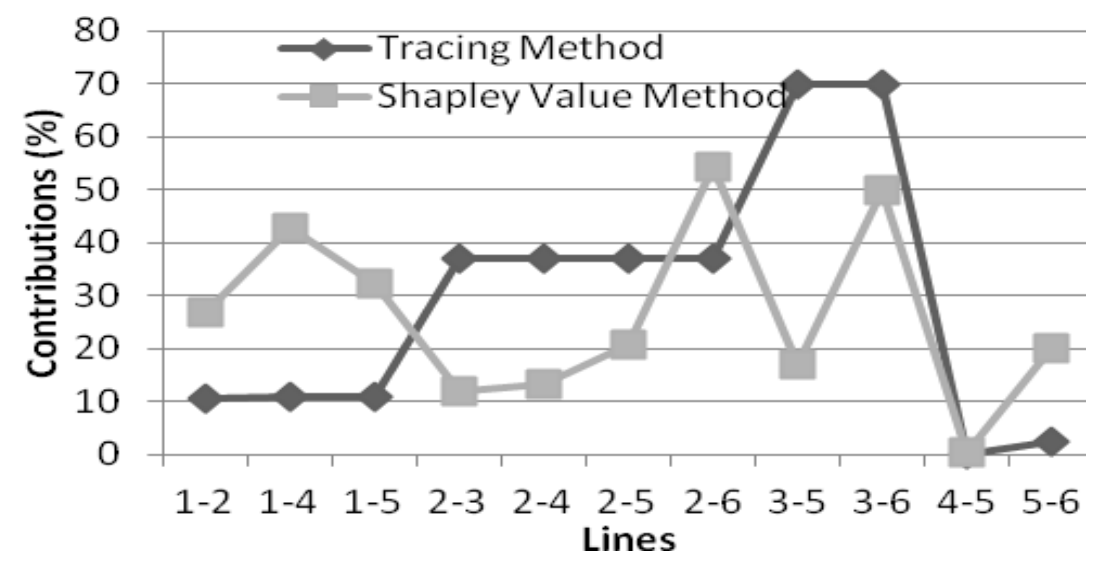

Figure 6 (c). Comparison of the usage allocation based on Tracing Method and Shapley Value Calculations to Load 6. 


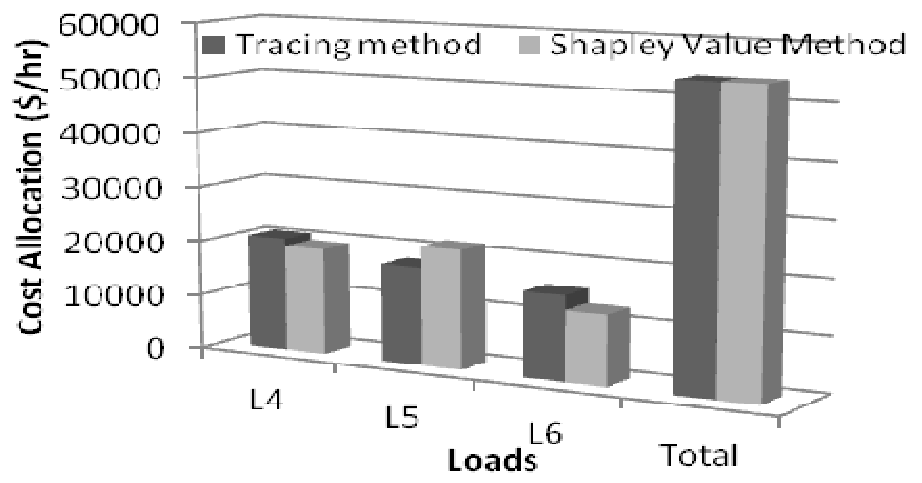

Figure 7. Comparison of the overall cost allocation based on Tracing Method and Shapley Value Calculations.

Figures 6 (a), (b) and (c). show the comparison of the usage allocation based on Tracing Method and Shapley Value Calculations to Load 4, Load 5 and Load 6 respectively.

Figure 7. shows the comparison of the overall cost allocation based on Tracing Method and Shapley Value Calculations.

\section{Conclusions}

In this paper, an easy and an efficient transmission pricing scheme using Shapley value method and power flow tracing method to allocate usage and cost of the transmission system in a deregulated environment is proposed. By comparing both the methods it is found that for a large power system allocation of usage and cost to users is difficult by using Shapley value method due to increase in number of coalitions. For proportional sharing method or power flow tracing method first it is necessary to trace the contributions of generators' and loads' to power flow and then these contributions are allocated to different players or users. From the case study it is shown that the proposed method is a simple, easy and direct method to allocate the transmission usage and cost to generators (and/or loads) in an open access electric market.

\section{APPENDIX}

Table 8 shows the line data of the sample 6 bus power system.

Table 8. Line data of the Sample 6 Bus Power System

\begin{tabular}{|l|l|l|l|}
\hline Line & $\begin{array}{l}\text { R } \\
\text { (p.u.) }\end{array}$ & $\begin{array}{l}\text { X } \\
\text { (p.u.) }\end{array}$ & $\begin{array}{l}\text { BL } \\
\text { (p.u.) }\end{array}$ \\
\hline $1-2$ & 0.10 & 0.20 & 0.04 \\
\hline $1-4$ & 0.05 & 0.20 & 0.04 \\
\hline $1-5$ & 0.08 & 0.30 & 0.06 \\
\hline $2-3$ & 0.05 & 0.25 & 0.06 \\
\hline $2-4$ & 0.05 & 0.10 & 0.02 \\
\hline $2-5$ & 0.10 & 0.30 & 0.04 \\
\hline $2-6$ & 0.07 & 0.20 & 0.05 \\
\hline $3-5$ & 0.12 & 0.26 & 0.05 \\
\hline $3-6$ & 0.02 & 0.10 & 0.02 \\
\hline $4-5$ & 0.20 & 0.40 & 0.08 \\
\hline $5-6$ & 0.10 & 0.30 & 0.06 \\
\hline
\end{tabular}


Electrical and Electronics Engineering: An International Journal (ELELIJ) Vol 3, No 3, August 2014

\section{REFERENCES}

[1] Daniel Kirschen and Goran Strbac, "Fundamentals of Power System Economics", Wiley Chichester, UK, 2004.

[2] M. Ilic, F.D. Galiana and L. Fink, "Power System Restructuring: Engineering and Economics, Norwell, MA, 1998.

[3] F.D. Galiana, A.J.Conjeo and H.A.Gill, "Transmission network cost allocation based on Equivalent Bilateral Exchnages, IEEE Trans Power System, vol 18, no 4, pp. 1425-1431, 2003.

[4] D.A. Lima et al., "An overview on network cost allcation methods", Electric Power Systems Research, vol 79, pp. 750-758, Dec 2008.

[5] D. Shrimohammadi et al., "Evaluation of transmission network capacity use for wheeling transactions," IEEE trans. On Power Systems, vol. 4, no. 4 pp. 1405-1413, Oct. 1989.

[6] D. Shrimohammadi et al. , "Cost of transmission transactions: An introduction," IEEE Trans. on Power Systems, vol. 6, no. 4 pp. 1546-1560, Nov. 1991.

[7] H.H. Happ, “ Cost of wheeling methodologies," IEEE Trans. on Power Systems, vol. 10,no. 2,pp. 1125 - 1142, May 1995.

[8] J.W. Marangon Lima, “ Allocation of transmission fixed charges:, An overview," IEEE Trans on Power Systems, vol. 11, no. 3, pp. 1409 - 1418, Aug 1996.

[9] R.R.Kovacs and A.L. Leverett, " A load flow based method for calculating embedded, incremental and marginal cost of transmission capacity,” IEEE Trans on Power Systems, vol. 9, no. 1, pp. 272 278, Feb 1994.

[10] B. Khan, G. Agnihotri and G. Gupta, "A Multipurpose Matrices Methodology for Transmission Usage, Loss and Reliability Margin Allocation in Restructured Environment", Electrical \& Computer Engineering: An International Journal (ECIJ) Vol 2, No 3, Sept. 2013.

[11] Tsukamoto, Y., and Iyoda, I., "Allocation of fixed transmission cost to wheeling transactions by cooperative game theory,” IEEE Trans. Power Syst., Vol. 11, No. 2, pp. 620-629, May 1996.

[12] Yu, C. W., David, A. K., and Wong, Y. K., "The use of game theory in transmission embedded cost allocation," 5th International Conferenceon Advancesin Power System Control, Operation and Management, pp. 139-143, Hong Kong, October 2000.

[13] Tan, X., and Lie, T. T., "Application of the Shapley value on transmission cost allocation in the competitive power market environment," IEE Proc. Generat. Transm. Distrib., Vol. 149, No. 1, pp. 15-20, January 2002.

[14] Zolezzi, J. M., and Rudnick, H., "Transmission cost allocation by cooperative games and coalition formation,” IEEE Trans. Power Syst., Vol. 17, No. 4, pp. 1008-1015, November 2002.

[15] Yu, C. W., David, A. K., Tse, C. T., and Chung, C. Y., "Capacity-use and reliability based transmission embedded cost allocation with temporal considerations," Int. J. Elect. Power Energy Syst., Vol. 25, pp. 201-208, 2003.

[16] Stamtsis, G. C., and Erlich, I., "Use of cooperative game theory in power system fixed-cost allocation,” IEE Proc. Generat. Transm. Distrib., Vol. 151, No. 3, pp. 401-406, May 2004.

[17] Happ, H. H., "Cost of wheeling methodologies," IEEE Trans. Power Syst., Vol. 9, No. 1, pp. 147156, February 1994. 2. Young, H. P., "Cost allocation," in Handbook of Game Theory Vol. 2, R. J. Aumann and S. Hart (Eds.), Amsterdam: Elsevier Science B. V., pp. 1193-1235, 2004.

[18] GeorgeA. Orfanos, PavlosS. Georgilakis and NikosD. Hatziargyriou, "A More Fair Power Flow Based Transmission Cost Allocation Scheme Considering Maximum Line Loading for N-1 Security”, IEEE Transactions on Power Systems, Vol.28, No.3, August2013.

[19] J. Bialek, D. B. Tam, "Tracing The Generators' Output”, Opportunities and Advances in International Power Generation, 18-20th March 1996.

[20] J. Bialek, "Topological Generation and Load Distribution Factors for Suppliment Charge Allocation in Transmission Open Access", 'I, IEEE Transactions on Power Systems, Vol. 12, No. 3, August 1997 pp, 1185-1193.

[21] Daniel Kirschen, Ron Allan, Goran Strbac, "Contributions of Individual Generators to Loads and Flows”, IEEE Transactions on Power Systems, Vol. 12, No. 1, February 1997.

[22] Ferdinand Gubina, David Grgi`c, Ivo Bani`, "A Method for Determining the Generators' Share in a Consumer Load", IEEE transactions on power systems, vol. 15, no. 4, November 2000.

[23] Felix F. Wu, Yixin Ni, and Ping Wei, "Power Transfer Allocation for Open Access Using Graph Theory - Fundamentals and Applications in Systems without Loop flow", IEEE transactions on power systems, vol. 15 , no. 3 , august 2000. 
[24] Kaigui Xie, Jiaqi Zhou, Wenyuan Li, "Analytical model and algorithm for tracing active power flow based on extended incidence matrix" Electric Power Systems Research 79 (2008) 399-405.

[25] Antonio J. Conejo, Javier Contreras, Delberis A. Lima, Antonio Padilha-Feltrin, "Z-bus Transmission Network Cost Allocation", IEEE transactions on power systems, vol. 22, no. 1, February 2007.

[26] A. R. Abhyankar, S. A. Soman, S. A. Khaparde, "Optimization Approach to Real Power Tracing: An Application to Transmission Fixed Cost Allocation”, IEEE transactions on power systems, vol. 21, no. 3, august 2006.

[27] Rao M.S.S., Soman S.A., Chitkara P., Gajbhiye R.K., Hemachandra N., Menezes B.L., "Min-Max Fair Power Flow Tracing for Transmission System Usage Cost Allocation: A Large System Perspective", IEEE Transactions on Power Systems, Vol. 25, no. 3, pp. 1457-1468, Aug. 2010.

[28] P. N. Biskas and A. G. Bakirtzis, "Decentralised security constrained DCOPFofinterconnectedpowersystems,"Proc.IEEGener.Transm. Distrib., vol. 151, no. 6, pp. 747-754, 2004.

[29] Silva E.L., Mesa S.E.C., Morozowski. M.: 'Transmission access pricing to wheeling transactions: a reliability based approach', IEEE Trans. Power Syst., 1998, 13, (4), pp. 1481-1486.

[30] D. Hur, C.-I. Yoo, B.H. Kim and J.-K. Park, "Transmission embedded cost allocation methodology with consideration of system reliability”, IEE Proc.-Gener. Transm. Distrib., Vol. 151, No. 4, July 2004.

[31] V. Vijay Venu, A. K. Verma, "A probabilistic transmission pricing methodology considering transmission reliability margins”, Int J Syst Assur Eng Manag (Apr-June 2010) 1(2):113-119.

[32] Monsef H, Jaefari M, "Transmission cost allocation based on use of reliability margin under contingency conditions”, IET Gener Transm, Distrib 3(6):574-585.Electronic Publication: Digital Object Identifiers (DOIs)

[33] K.-H. Chung, B. H. Kim, D. Hur, and J.-K. Park, "Transmission re- liability cost allocation method based on market participants' reliability contribution factors,"Electr.PowerSyst.Res.,vol.73,no.1,pp. 31-36, Jan.

[34] Z.Jing,X.Duan,F.Wen,Y.Ni,andF.F.Wu,"Review of transmission fixed cost allocation methods,"inProc. IEEE Power Eng. Soc.General Meeting, Toronto, on, Canada, 2003.

[35] R. D. Christie, B. F. Wollenberg, and I. Wangensteen, "Transmission management in the deregulated environment,’Proc.IEEE,vol.88,no. 2, pp. 170-195, Feb. 2005.

[36] Merrill, H.M., and Erickson. B.W.: 'Wheeling rates based on marginalcost theory', IEEE Trans. Power System, vol 4, no 4, pp. 1445-1451, October 1989.

[37] N.B.Dev Choudhury and S. K. Goswami, “ Transmission loss allocation using combined game theory and artificial neural network", International journal Electrical Power and Electric Systems, vol 43, pp. 554-561, July, 2012.

[38] R. Bhakar et al., "Transmission Embedded Cost Allocation in Restructured Environment: A Gametheoretic Approach", Electric Power Components and Systems, vol 37, pp. 970-981, 2009.

[39] J. Nikoukar, M.R. Haghifam, " Transmission cost allocation based on the use of system and considering the congestion cost”, Electrical Power and Energy Systems, vol 43, pp. 961-968, July 2012.

[40] Allen. J. Wood and Bruce F. Wollenberg,"Power Generation, Operation, and Control, 2nd Edition", John Wiley \& Sons, NY, Jan 1996Lee, S.hyun. \& Kim Mi Na, (2008).

\section{Authors}

Pawan Rathore was born in Balco Town, Korba City, Chhattisgarh State, India in 1990. He received the B.E. degree in Electrical and Electronics engineering from the Chhattisgarh Swami Vivekananda Technical University, Bhilai in 2012. Currently, he is pursuing M.Tech from Maulana Azad National Institute of Technology, Bhopal. His research interests include power system optimization, congestion management, transmission pricing and application of artificial intelligence techniques in power system.

Ganga Agnihotri received BE degree in Electrical engineering from MACT, Bhopal (1972), the ME degree (1974) and PhD degree (1989) from University of Roorkee, India. Since 1976 she is with Maulana Azad College of Technology, Bhopal in various positions. Currently she is professor. Her research interest includes Power System Analysis, Power System Optimization and Distribution Operation.
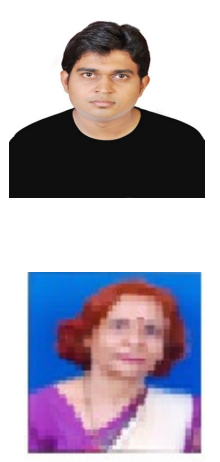
Baseem Khan was born in Gwalior, India in 1987. He received BE degree (2008) from Maharana Pratap College of Technology Gwalior and received an M.Tech. degree (2010) in Power System from MANIT Bhopal. At the moment he is a research scholar at MANIT Bhopal, India. His research interests include power system optimization, congestion management, transmission pricing and application of artificial intelligence techniques in power system.

Garima Naidu was born in Bhopal, Madhya Pradesh State, India in 1989. She received her B.Tech degree in Electrical and Electronics engineering from Vellore Institute of Technology, Vellore, Tamil Nadu in 2012. Currently, she is pursuing M.Tech from Maulana Azad National Institute of Technology, Bhopal. Her research interests include power system optimization, FACTS devices, and application of artificial intelligence techniques in power system.
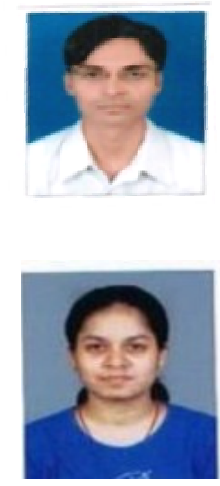